\title{
Quality of life, psychosocial health, and antiretroviral therapy among HIV-positive women in Zimbabwe
}

\section{Citation}

Patel, Rena, Seble Kassaye, Cheryl Gore-Felton, Grace Wyshak, Gerard Kadzirange, Godfrey Woelk, and David Katzenstein. 2009. Quality of life, psychosocial health, and antiretroviral therapy among HIV-positive women in Zimbabwe. AIDS Care 21, no. 12: 1517-1527. doi:10.1080/09540120902923055.

\section{Published Version}

doi:10.1080/09540120902923055

\section{Permanent link}

http://nrs.harvard.edu/urn-3:HUL.InstRepos:28993653

\section{Terms of Use}

This article was downloaded from Harvard University's DASH repository, and is made available under the terms and conditions applicable to Other Posted Material, as set forth at http:// nrs.harvard.edu/urn-3:HUL.InstRepos:dash.current.terms-of-use\#LAA

\section{Share Your Story}

The Harvard community has made this article openly available.

Please share how this access benefits you. Submit a story.

Accessibility 
Published in final edited form as:

AIDS Care. 2009 December ; 21(12): 1517-1527. doi:10.1080/09540120902923055.

\title{
Quality of life, psychosocial health, and antiretroviral therapy among HIV-positive women in Zimbabwe
}

\author{
Rena Patel ${ }^{a,}{ }^{,}$, Seble Kassaye ${ }^{b}$, Cheryl Gore-Felton $^{c}$, Grace Wyshak $^{\mathrm{d}}$, Gerard Kadzirange ${ }^{\mathrm{e}}$, \\ Godfrey Woelk ${ }^{\dagger}$, and David Katzenstein ${ }^{b}$ \\ aDepartment of Internal Medicine, Stanford University, Palo Alto, CA, USA \\ bDepartment of Infectious Diseases, Stanford University School of Medicine, Palo Alto, CA, USA \\ 'Department of Psychiatry \& Behavioral Science, Stanford University School of Medicine, Palo \\ Alto, CA, USA \\ ${ }^{d}$ Department of International Health, School of Public Health, Harvard University, Boston, MA, \\ USA
}

eZimbabwe AIDS Prevention Project (ZAPP), Harare, Zimbabwe

fDepartment of Community Health, University of Zimbabwe, Harare, Zimbabwe

\section{Abstract}

Little is known about the psychosocial impact of antiretroviral therapy (ART) among women in sub-Saharan Africa. Therefore, we conducted a cross-sectional study in Zimbabwe to assess the impact of ART on HIV-positive women's health-related quality of life, using the Medical Outcomes Study-HIV Quality of Life (QOL) questionnaire. Additionally, we assessed sociodemographics, reproductive and sexual health, HIV-related history, disclosure, social stigma, selfesteem, and depression. Structured interviews were conducted with $200 \mathrm{HIV}$-positive women and categorized into three groups by treatment: (1) Group $1(n=31)$ did not meet clinical or laboratory criteria to begin treatment; (2) Group $2(n=73)$ was eligible to begin treatment but awaiting initiation of treatment; and (3) Group $3(n=96)$ was on ART for a median of 13 months. The women had similar sociodemographic characteristics but varied significantly in clinical characteristics. Women on ART reported fewer AIDS-related symptoms in the last week and year and had higher current and lower baseline CD4 counts compared to women not on ART. On most QOL domains women on ART reported higher mean scores as compared to women not on ART $(p<0.01)$. Additionally, women on ART reported less depression compared to women not on ART $(p<0.001)$. Between the two groups of women not on ART, unexpectedly, there were no significant differences in their scores for QOL or depression. Thus, Zimbabwean women living with HIV experience better overall QOL and lower depression on ART. Altogether, our findings suggest that ART delivery in resource-poor communities can enhance overall QOL as well as psychosocial functioning, which has wide-ranging public health implications.

(C) 2009 Taylor \& Francis

*Corresponding author. rcpatel@ stanford.edu. 


\section{Keywords}

quality of life; depression; HIV treatment; sub-Saharan Africa; Zimbabwe

\section{Introduction}

In Zimbabwe, an estimated 15.6\% of adults are living with HIV/AIDS (UNGASS, 2008), reducing life expectancy to 34 years for women (World Health Organization, 2005).

Increasing access to antiretroviral therapy (ART) offers hope. In 2007, approximately $20 \%$

of infected women needing ART in Zimbabwe were receiving treatment. Although the longterm goal of ART is to restore health and improve quality of life (QOL) for people living with HIV/AIDS (PLWHA), little is known about the effects of ART on QOL and psychosocial health among women, particularly in sub-Saharan Africa (SSA).

\section{HIV and quality of life (QOL)}

Health-related quality of life (HR-QOL) is a multidimensional construct for patients' perceived well-being, centered on physical, mental, and social functioning (Smith, Avis, \& Assmann, 1999). Several measures capture HR-QOL (Wu, 2000) and changes in QOL in PLWHA initiating ART has been studied in Western settings (Jia, Uphold, Wu, Chen, \& Duncan, 2005; Liu et al., 2006; Murri et al., 2003). Nonetheless, which socio-economic, psychosocial, and clinical factors mediate perceived HR-QOL in PLWHA is unclear.

Several studies have demonstrated the feasibility and efficacy of ART in resource-poor settings (Djomand et al., 2003; Laurent et al., 2005; Sow et al., 2007) but only recently have studies focused on HR-QOL in patients on treatment (Ichikawa \& Natpratan, 2006; Jelsma, Maclean, Hughes, Tinise, \& Darder, 2005; Poupard et al., 2007; Stangl, Wamai, Mermin, Awor, \& Bunnell, 2007). In South Africa, Jelsma et al. (2005) demonstrated a significant increase in HR-QOL among HIV-positive patients after 12 months of ART in a public sector program. The most comprehensive HR-QOL research in SSA by Stangl et al. (2007) prospectively followed 947 AIDS patients initiating treatment in rural Uganda. At study entry depression, lower CD4 counts, higher HIV RNA levels, economic dependence, and lower education predicted lower initial HR-QOL but economic dependence was the sole predictor of lower HR-QOL at follow-up (Stangl et al., 2007).

Limited resources require prioritizing access to ART. QOL measures may add to biologic measures of ART response to assess resource allocation and health outcomes. Here, we examined socio-economic, psychosocial, and clinical measures among HIV-positive women living in Zimbabwe who were at different stages of initiating ART. We studied women because they constitute more than $80 \%$ of the ART recipients, consistent with the overrepresentation of women in treatment programs in Zimbabwe and generally in SSA (UNAIDS, 2008). 


\section{Methods}

\section{Participants}

Women were recruited between June and August 2007 at the opportunistic infections (OI) clinic at the Chitungwiza Regional Hospital, a national ART rollout site $25 \mathrm{~km}$ from Harare (Figure 1). Study eligibility included age $>18$ years, HIV infection, being on ART or on the "waiting list," and ability to provide informed consent. Individuals enrolled in another study or who did not speak English, Shona, or Ndebele were excluded.

After informed consent was obtained, interviewers administered a one-hour questionnaire. Study nurses assessed participants' current HIV-related symptoms and illnesses, and obtained blood samples. Laboratory results were provided to the participants and included in their medical records. The Stanford University Institutional Review Board and the Medical Research Council of Zimbabwe approved the study.

\section{Measures}

Socio-demographics, reproductive and sexual health, HIV-related history, disclosure, social stigma, self-esteem, depression, and QOL were assessed. "Baseline clinical data" including initial CD4 counts, WHO clinical staging, and duration on ART were obtained through chart review. The questionnaire was translated into Shona and back-translated into English. Three female interviewers were selected from the community and trained.

Disclosure-We modified the disclosure matrix in the Bauman Decision to Disclose Scale (Pequegnat et al., 2001) by adding more targets and choices on how they reacted to the disclosure.

Social stigma-We modified the 40-question HIV Stigma Scale (Berger, Ferrans, \& Lashley, 2001) to include 11 questions in total and divided the scale into perceived and experienced stigma. Items were summed for a total score and higher scores indicate lower stigma. The Cronbach's coefficient alphas for the two subscales were 0.80 (stigma perceived) and 0.74 (stigma experienced).

Self-esteem-The self-esteem scale was adapted for local context from the University of California at San Francisco Center for AIDS Prevention Studies (UCSF CAPS) HIV Counseling and Testing questionnaire (Coates et al., 2000). Higher scores indicate higher self-esteem. The Cronbach's coefficient alpha was 0.76 for the scale.

Depression-The depression scale was adapted for local context from the UCSF CAPS HIV Counseling and Testing questionnaire validated in developing countries (Coates et al., 2000). A second depression scale, the Shona Symptom Questionnaire (SSQ14) was used, which has been developed and validated among HIV-positive Zimbabweans (Nhiwatiwa, Patel, \& Acuda, 1998; Patel, Simunyu, Gwanzura, Lewis, \& Mann, 1997; Patel et al., 1998; Stranix-Chibanda et al., 2005). For both scales, higher scores indicate worse depressive symptoms. Questions about somatic symptoms were removed from analysis to avoid conflating symptoms of HIV disease with those of depression (Kalichman, Rompa, \& Cage, 2000). A second scale was used to further validate the SSQ14; the SSQ14 was the preferred 
depression measurement tool because it was developed locally and thus culturally relevant. The Cronbach's coefficient alphas were 0.75 for the depression scale and 0.86 for the SSQ14 and the total scores on the two scales had an alpha of 0.80 .

Quality of life (QOL)—The Medical Outcomes Study-HIV (MOS-HIV) QOL questionnaire (Wu, Revicki, Jacobson, \& Malitz, 1997) has been validated in Zimbabwe (Taylor, Dolezal, Tross, \& Holmes, 2009) and other sub- Saharan settings such as Uganda (Mast et al., 2004). The instrument contains 11 subscales or domains of HR-QOL, including health perceptions, pain, physical functioning, role functioning, social functioning, mental health, vitality, health distress, cognitive functioning, health transition, and overall QOL. To impute missing values in subscales, averages were used when more than half the values were present; only six $(0.001 \%)$ single values were missing for the MOS-HIV QOL data which were imputed as described above. The subscale scores were then transformed on a 0 100 scale to have a mean of 50 and a standard deviation of 10, with higher scores representing better QOL (Revicki, Sorensen, \& Wu, 1998). Factor analysis was used to construct summary scores of the MOS-HIV QOL questionnaire for physical and mental health (Revicki et al., 1998). The inter-reliability of the subscales was assessed using Cronbach's coefficient alpha, which ranged from 0.62 (mental health) to 0.92 (health distress).

CD4 counts-Current CD4 T-lymphocytes were enumerated using Guava Easy-CD4 (Guava Technologies, Hayward, CA) or BD FACSCount ${ }^{\mathrm{TM}}$ (Becton Dickinson, Mountain View, CA).

\section{Data analysis}

The study was designed to have $80 \%$ power with an alpha of 0.05 to detect a $15 \%$ increase in CD4 counts from baseline for the patients on ART. All statistical analyses were carried out using SAS version 9.1 (Cary, North Carolina). Chi-square $\left(\chi^{2}\right)$ or Fisher's exact tests were used to test categorical variables and ANOVA for continuous variables. A general linear model was used to adjust for confounding variables and a linear regression model to identify predictors of better QOL.

Certain continuous variables, such as household goods, number of people disclosed to, selfreported signs and symptoms in the last year, duration of HIV, and SSQ14 were transformed into binary measures based on median cut-offs except for age, education, and CD4 levels.

The participants were classified into three groups (Figure 1) according to eligibility and receipt of ART. The eligibility criteria for starting treatment were based on WHO 2003 recommendations when CD $4 \leq 200$ cells $/ \mathrm{mm}^{3}$ or at WHO stage 3 or 4 disease (World Health Organization, 2004). The first group of women $(n=31)$ were not eligible for treatment based on clinical or laboratory criteria. The second group $(n=73)$ were eligible for but not receiving ART, largely due to limited drug access. The third group $(n=96)$ were receiving ART. Therefore, Group 1 is termed INELIGIBLE, Group 2 WAITING, and Group 3 TREATED groups. CD4 counts used for classification were based on the values obtained by the study and the WHO clinical staging criteria were recorded in the patients' medical charts at baseline. 


\section{Results}

The median age of the women was 35 years (range 22-69 years). Seventy percentage had completed at least some secondary schooling and nearly all were literate. Thirty seven percentage were currently married and $45 \%$ reported being unemployed, which excluded self or informal employment. Approximately $47 \%$ of the women reported ever using a condom, and their median number of pregnancies was three with median age of sexual debut at 19 years. Women reported a median of two lifetime sexual partners and one in the past year. The median baseline and current CD4 counts were 176 and 254 cells $/ \mathrm{mm}^{3}$, respectively, and $60 \%$ of the women reported a history of WHO clinical stage 3 or 4 disease.

The three groups of women were similar in baseline socio-demographic characteristics (Table 1). However, the mean age for the treated group of 36.4 years (median 37) was significantly greater than those for the ineligible and waiting groups of women, 35.2 and 34.2 (median 34 and 33) years, respectively ( $p=0.0029$ ).

Clinical characteristics (Table 2), differed among the three groups. Baseline CD4 counts were greater in the ineligible and waiting groups compared to the treated group (medians Group $1=386$, Group $2=353$, and Group $3=176 ; p<0.0001$ ). In contrast, current CD4 counts were comparable between the ineligible and treated groups and lowest among the awaiting group (medians Group $1=315$, Group $2=145$, and Group $3=327 ; p<0.0001$ ). A high proportion of the treated (70\%) and waiting groups (74\%) had a WHO clinical stage 3 or 4 diagnosis which varied significantly between the three groups $(p<0.0001)$. The mean number of symptoms reported at baseline were highest in the treated group and lowest in the ineligible group ( $p=0.0001$ ). This trend reversed at the time of the study as the ineligible group reported the highest number of symptoms $(p<0.0001)$. The mean number of current AIDS-related symptoms and illnesses reported were higher and comparable between the ineligible (5.8) and waiting groups (6.0) and lower in the treated group (5.0) $(p=0.053)$.

\section{Quality of life (QOL) trends}

For all MOS-HIV QOL domains, except social function, mean scores were highest, indicating best QOL, in the treated group (Table 3). Eight of the 11 mean domain scores were significantly different for the three groups: health perceptions, bodily pain, role function, vitality, health distress, cognitive function, QOL, and health transitions (all at $p<$ 0.0001 except for role function $p=0.01$ ). Physical function and mental health domain scores were not significantly different for the three groups although there was a trend toward higher scores in the treated group. The overall mean score for the entire questionnaire was significantly highest in the treated group ( $p<0.0001)$. The mean mental health summary (MHS) score was the highest in the treated group $(p<0.0001)$. The mean physical health summary (PHS) score was not statistically different for the three groups $(p<0.74)$.

On multivariate analysis (Table 3) all of the unadjusted QOL domain scores except for role function remained significantly better in the treated group (all $p<0.05$ ). The mean and MHS adjusted scores were again significantly better in the treated group $(p<0.0001)$. Adjusted PHS scores remained insignificantly different in the three groups. 


\section{Psychosocial measures}

Psychosocial measures were most positive in the treated group (Table 4). Women on ART scored most favorably on the UCSF CAPS depression scale with a mean score of 14.32 out of 28, while the ineligible group scored 17.87 and the waiting group 16.70 ( $p=0.002$ ). With the SSQ14, similarly, the treated group had a mean score of 26.01 out of 48 while the other two groups scored approximately 30 each $(p=0.002)$. The median number of people a woman disclosed her HIV status to was five in the treated group, three in the ineligible and waiting groups ( $p=0.0006$ ). The groups scored similarly on the perceived social stigma scale but did differ slightly with their scores on the experienced social stigma scale, with the groups scoring a mean of 13.94, 14.90, and 15.98 out of a possible 24 for Groups 1, 2, and 3, respectively ( $p=0.05$ ). Their mean scores on the self-esteem scale did not differ significantly though the treated group had the highest mean score. A multivariate analysis revealed the treated group had the most favorable scores on the depression and SSQ14 scales (both $p<0.001$; Table 4).

\section{Quality of life (QOL) and CD4 count as functions of duration of antiretroviral therapy (ART)}

Among the women on ART there is a non-significant trend toward increased QOL with increasing duration of ART (Figures 2a,b, $p>0.05$ ). However, there is a more robust correlation between change in CD4 counts and treatment duration (Figure 3) with the Spearman correlation coefficient for change in CD4 count, current minus baseline, and duration on ART of $0.55(p<0.0001)$.

\section{Predictors of Quality of life (QOL)}

A step-wise linear regression model was carried out with the three different summary scores of QOL: overall mean, PHS, and MHS (Table 5). Treatment, higher education, lower symptoms and illnesses in the last year, higher CD4 count, lower WHO clinical stage, and lower depressive symptoms were significantly associated with better mean QOL score. Only lower symptoms and illnesses and lower WHO clinical stage were significantly associated with better PHS. Treatment, greater number of household goods, higher CD4 count, and lower depressive symptoms were significantly associated with better MHS.

\section{Discussion}

Our study findings indicate that women on ART had better QOL than women not on ART, consistent with prior studies in Africa (Jelsma et al., 2005; Stangl et al., 2007). As expected, CD4 counts improved and symptoms abated on ART and the treated women had significantly lower depressive symptoms. The effect of treatment on QOL remained after adjusting for socio-economic status (SES; age, education, household goods, and religion), clinical (number of symptoms, CD4 count, and clinical stage), and psychosocial factors (depression and number of people disclosed to). Stangl et al. (2007) found economic dependence predictive of QOL at follow-up, which was not directly measured in our cohort. Nonetheless, our study population's socio-economic homogeneity may have reduced our ability to assess the impact of SES on QOL. Generally speaking, aside from viral load, which was not measured in our study, it is unlikely that other clinical variables impact QOL. Therefore, there are likely other psychosocial factors, such as hopefulness or fear, which 
mediate the association between treatment and perceived QOL. In addition, that the social function domain remained constant across the three groups may imply that social connectivity in this community is independent of health status.

Treatment is more robustly associated with mental health, while disease status is more closely associated with physical health. Significant predictors of PHS scores were number of self-endorsed AIDS-related sign and symptoms and baseline WHO clinical staging, not treatment itself. The MHS scores were highest and the levels of depression lowest in the treated group compared to ineligible and waiting groups, both unadjusted and adjusted. As previously noted, we did not find a significant difference on PHS scores between treated and untreated women. Treatment may, therefore, more rapidly improve mental health than physical health. Women on treatment may also have greater expectations of being well and expect more explicit gains in physical health. Thus, despite lower symptoms and higher current CD4 counts, the treated women perceive their health to be relatively worse than the untreated women. Additionally, treatment may have negative effects on PHS due to its side effects and toxicities. Lastly, patients are known to emphasize mental health rather than physical functioning on QOL questionnaires (Smith et al., 1999).

Factors predictive of better QOL scores were: treatment; clinical factors (e.g., fewer AIDSrelated symptoms, higher CD4 counts, and lower clinical staging); and psychosocial factors (e.g., lower depression). Socio-economic factors (e.g., higher education and greater number of household goods) predicted higher QOL scores in only one of the three summary scores. Therefore, we cannot generalize the significance of these factors' impact to all of the domains of QOL. Consistent with our findings, Stangl et al. (2007) found similar clinical, socio-economic, and psychosocial factors predictive of better QOL. A reason for our limited ability to detect other SES and psychosocial predictors may reflect the sociocultural context in which we conducted the study (i.e., the homogeneity of our study population). Thus, future studies need to include QOL assessments that are culturally tailored to the Zimbabwean context, enroll socially heterogeneous groups, and include men to further assess the influence of socio-economic factors on QOL in Zimbabwe.

We report a unique finding that the QOL scores were similar for HIV-positive women ineligible for treatment and those with AIDS awaiting treatment. We anticipated lower QOL scores among women with AIDS awaiting treatment compared to those without AIDS and those already on treatment. However, the two groups of women not receiving ART had very similar QOL as well as depression scores, despite the significant differences in symptoms and CD4 counts. One explanation for this finding may be that women with HIV are vulnerable to social and individual stressors that impact their mental and physical health, regardless of differences in symptoms and CD4 counts. Moreover, tenuous access to ART in a struggling economy may impact psychosocial health and therefore overall QOL, particularly among the ineligible women. For instance, they may feel ignored or unfairly treated. Regardless, all HIV-positive women require social support and programming to address their QOL and psychosocial needs. Additionally, the finding of marked improvement in QOL and mental health associated with treatment argues for greater access to ART. In fact, CD4 levels which trigger treatment have recently been raised in the USA (Department of Health and Human Services, 2008) and many resource-limited countries are 
considering changing their guidelines to provide treatment for HIV-infected individuals with $\leq 350 \mathrm{CD} 4$ cells $/ \mathrm{mm}^{3}$. Fifty-five percentage of the ineligible women in our study had a CD4 count less than 350 cells $/ \mathrm{mm}^{3}$ at study entry and we welcome the prospect of broader access to earlier treatment in order to improve HR-QOL.

The aggregate QOL scores of the Zimbabwean women in this study are lower than those of other cohorts in SSA. For example, Stangl et al. (2007) found that the baseline QOL scores ranged from 20 to 66 and the 12-month follow-up scores ranging from 58 to 94 . Scores in our waiting group ranged between 17-69 and 38-66 in the treated group. The lower scores in these Zimbabwean women may highlight the social, economic, and political hardships they currently confront. Taylor et al.'s (2009) cohort of rural Zimbabwean men and women with presumed HIV infection had domain mean scores similar to ours, ranging from 30 to 62.

\section{Strengths and limitations of study}

This study adds to the growing literature on psychosocial impact of ART. Psychosocial measures such as depression, disclosure, stigma, self-esteem, and QOL were assessed with standardized, validated tools, with a reasonable sample size.

While the treated group demonstrated higher QOL scores compared to those not yet on treatment, we cannot ascertain if higher scores were due to treatment given the study's cross-sectional design. The addition of focus groups or in-depth interviews may yield more information on psychosocial factors impacting QOL. The convenience sample may have introduced a healthy cohort bias of the women being healthy enough to attend the clinic; conversely, others presented with complaints. Some women may have over-reported their symptoms or negative experiences in the hopes of receiving more medical attention. For the women in the ineligible and waiting groups, our study also did not capture their interpretation of the gravity of their HIV disease, their perceived likelihood of getting treatment, or their number on the "waitlist." These factors may better explain the role of hopefulness and self-perception on psychosocial health.

\section{Future directions}

This study highlights the need for further research on QOL out comes in various groups of HIV-positive individuals, especially those who are ineligible or awaiting treatment. Our findings suggest QOL benefits and depression reduction with ART. Qualitative studies are needed to better characterize the psychosocial mediators of ART's impact on QOL.

Knowing whether the trends observed in our study hold true in other sub-Saharan African settings would help in prioritizing resources and developing policies.

\section{Acknowledgments}

We acknowledge foremost the Zimbabwean women's participation in this project. Thank you to the staff at Zimbabwean AIDS Prevention Project (ZAPP) and Tessa Andermann for providing support during study implementation. Thank you to Dr. Mark Vosvick for providing guidance on the psychosocial measures and Dr. Albert Wu on analyzing the quality of life questionnaire.

The study was supported by the Medical Scholars Fellowship at Stanford University School of Medicine, National Institutes of Health (NIAID R01 AI060399), and the Fogarty International Center (ICOHRTA U2R TW006878). 


\section{References}

Berger BE, Ferrans CE, Lashley FR. Measuring stigma in people with HIV: Psychometric assessment of the HIV stigma scale. Research in Nursing and Health. 2001; 24(6):518-529. [PubMed: 11746080]

Coates TJ, Grinstead OA, Gregorich SE, Sweat MD, Kamenga MC, Sangiwa G, et al. Efficacy of voluntary HIV-1 counselling and testing in individuals and couples in Kenya, Tanzania, and Trinidad: A randomised trial. Lancet. 2000; 356(9224):103-112. [PubMed: 10963246]

Department of Health and Human Services. Panel on antiretroviral guidelines for adults and adolescents. Guidelines for the use of antiretroviral agents in HIV-1-infected adults and adolescents. Panel on antiretroviral guidelines for adults and adolescents. Guidelines for the use of antiretroviral agents in HIV-1-infected adults and adolescents. 2008. http://www.aidsinfo.nih.gov/ ContentFiles/AdultandAdolescentGL.pdf

Djomand G, Roels T, Ellerbrock T, Hanson D, Diomande F, Monga B, et al. Virologic and immunologic outcomes and programmatic challenges of an antiretroviral treatment pilot project in Abidjan, Cote d'Ivoire. AIDS. 2003; 17(Suppl 3):S5-S15. [PubMed: 14565604]

Ichikawa M, Natpratan C. Perceived social environment and quality of life among people living with HIV/AIDS in northern Thailand. AIDS Care. 2006; 18(2):128-132. [PubMed: 16338770]

Jelsma J, Maclean E, Hughes J, Tinise X, Darder M. An investigation into the health-related quality of life of individuals living with HIV who are receiving HAART. AIDS Care. 2005; 17(5):579-588. [PubMed: 16036244]

Jia H, Uphold CR, Wu S, Chen GJ, Duncan PW. Predictors of changes in health-related quality of life among men with HIV infection in the HAART era. AIDS Patient Care and STDS. 2005; 19(6):395405. [PubMed: 15989435]

Kalichman SC, Rompa D, Cage M. Distinguishing between overlapping somatic symptoms of depression and HIV disease in people living with HIV-AIDS. Journal of Nervous and Mental Disease. 2000; 188(10):662-670. [PubMed: 11048815]

Laurent C, Ngom Gueye NF, Ndour CT, Gueye PM, Diouf M, Diakhate N, et al. Long-term benefits of highly active antiretroviral therapy in Senegalese HIV-1-infected adults. Journal of Acquired Immune Deficiency Syndromes. 2005; 38(1):14-17. [PubMed: 15608518]

Liu C, Johnson L, Ostrow D, Silvestre A, Visscher B, Jacobson LP. Predictors for lower quality of life in the HAART era among HIV-infected men. Journal of Acquired Immune Deficiency Syndromes. 2006; 42(4):470-477. [PubMed: 16810114]

Mast TC, Kigozi G, Wabwire-Mangen F, Black R, Sewankambo N, Serwadda D, et al. Measuring quality of life among HIV-infected women using a culturally adapted questionnaire in Rakai district, Uganda. AIDS Care. 2004; 16(1):81-94. [PubMed: 14660146]

Murri R, Fantoni M, Del Borgo C, Visona R, Barracco A, Zambelli A, et al. Determinants of healthrelated quality of life in HIV-infected patients. AIDS Care. 2003; 15(4):581-590. [PubMed: 14509872]

Nhiwatiwa S, Patel V, Acuda W. Predicting postnatal mental disorder with a screening questionnaire: A prospective cohort study from Zimbabwe. Journal of Epidemiology and Community Health. 1998; 52(4):262-266. [PubMed: 9616415]

Patel V, Simunyu E, Gwanzura F, Lewis G, Mann A. The Shona symptom questionnaire: The development of an indigenous measure of common mental disorders in Harare. Acta Psychiatrica Scandinavica. 1997; 95(6):469-475. [PubMed: 9242841]

Patel V, Todd C, Winston M, Gwanzura F, Simunyu E, Acuda W, et al. Outcome of common mental disorders in Harare, Zimbabwe. British Journal of Psychiatry. 1998; 172:53-57. [PubMed: 9534833]

Pequegnat W, Bauman LJ, Bray JH, DiClemente R, Dilorio C, Hopper SK, et al. Measurement of the role of families in prevention and adaptation to HIV/AIDS. AIDS and Behavior. 2001; 5(1):1-19.

Poupard M, Ngom Gueye NF, Thiam D, Ndiaye B, Girard PM, Delaporte E, et al. Quality of life and depression among HIV-infected patients receiving efavirenz- or protease inhibitor-based therapy in Senegal. HIV Medicine. 2007; 8(2):92-95. [PubMed: 17352765] 
Revicki DA, Sorensen S, Wu AW. Reliability and validity of physical and mental health summary scores from the Medical Outcomes Study HIV Health Survey. Medical Care. 1998; 36(2):126137. [PubMed: 9475468]

Smith KW, Avis NE, Assmann SF. Distinguishing between quality of life and health status in quality of life research: A meta-analysis. Quality of Life Research. 1999; 8(5):447-459. [PubMed: 10474286]

Sow PS, Otieno LF, Bissagnene E, Kityo C, Bennink R, Clevenbergh P, et al. Implementation of an antiretroviral access program for HIV-1-infected individuals in resource-limited settings: Clinical results from 4 African countries. Journal of Acquired Immune Deficiency Syndromes. 2007; 44(3):262-267. [PubMed: 17146376]

Stangl AL, Wamai N, Mermin J, Awor AC, Bunnell RE. Trends and predictors of quality of life among HIV-infected adults taking highly active antiretroviral therapy in rural Uganda. AIDS Care. 2007; 19(5):626-636. [PubMed: 17505923]

Stranix-Chibanda L, Chibanda D, Chingono A, Montgomery E, Wells J, Maldonado Y, et al. Screening for psychological morbidity in HIV-infected and HIV-uninfected pregnant women using community counselors in Zimbabwe. Journal of the International Association of Physicians AIDS Care (Chicago, IL). 2005; 4(4):83-88.

Taylor T, Dolezal C, Tross S, Holmes WC. Reliability and validity of two HIV/AIDS-specific quality of life instruments adapted for use in HIV-positive Zimbabweans. AIDS Care. 2009; 21(5):598607. [PubMed: 19444668]

UNAIDS. Report on the global AIDS epidemic. 2008. Retrieved March 3, 2009, from http:// www.unaids.org/en/KnowledgeCentre/HIVData/GlobalReport/2008/2008_Global_report.asp

UNGASS. UNGASS report on HIV and AIDS: Zimbabwe country report. 2008. Retrieved February 15, 2009, from http://data.unaids.org/pub/Report/2008/ zimbabwe_2008_country_progress_report_en.pdf

World Health Organization. Scaling up antiretroviral therapy in resource-limited settings: Treatment guidelines for a public health approach. 2004. Retrieved March 3, 2009, from http:// www.who.int/hiv/pub/prev_care/en/arvrevision2003en.pdf

World Health Organization. Profile on HIV/AIDS scale-up: Zimbabwe. 2005. Retrieved February 15, 2009, from http://www.who.int/hiv/HIVCP_ZWE.pdf

Wu AW. Quality of life assessment comes of age in the era of highly active antiretroviral therapy. AIDS. 2000; 14(10):1449-1451. [PubMed: 10930161]

Wu AW, Revicki DA, Jacobson D, Malitz FE. Evidence for reliability, validity and usefulness of the Medical Outcomes Study HIV Health Survey (MOS-HIV). Quality of Life Research. 1997; 6(6): 481-493. [PubMed: 9330549] 


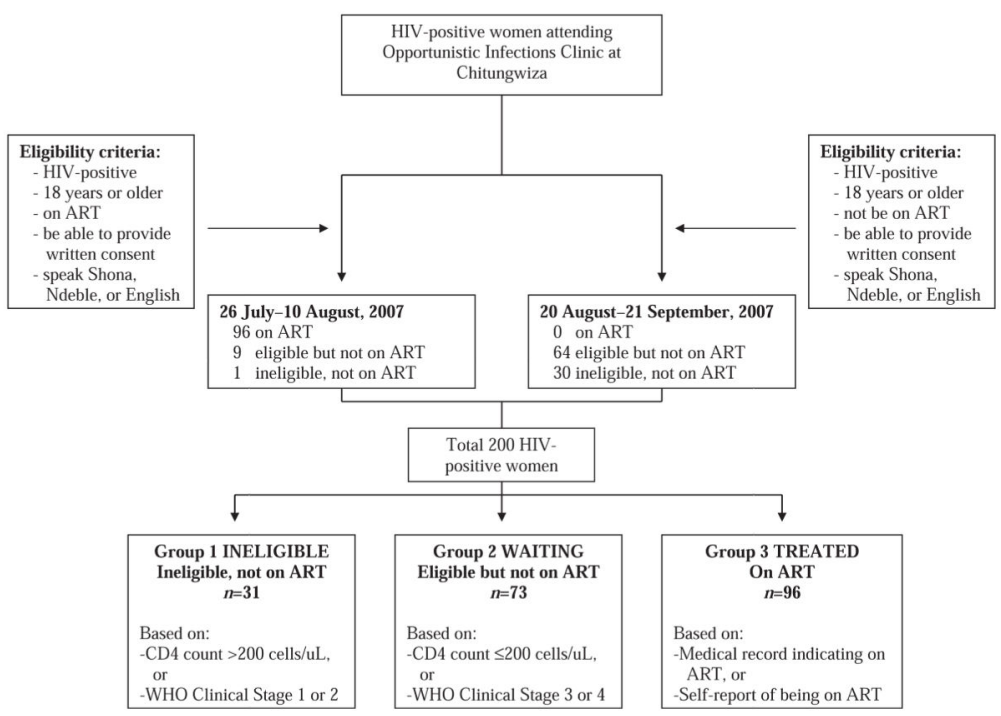

Figure 1.

Participant recruitment. 
(a)

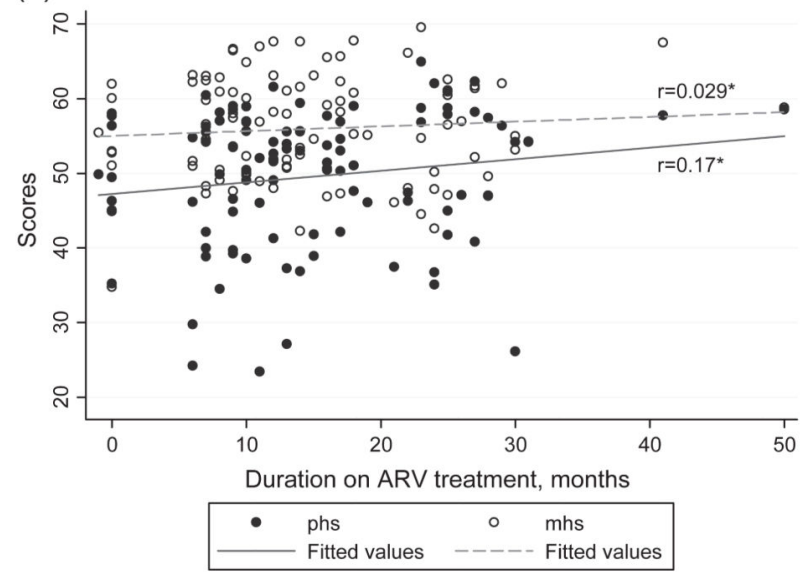

(b)

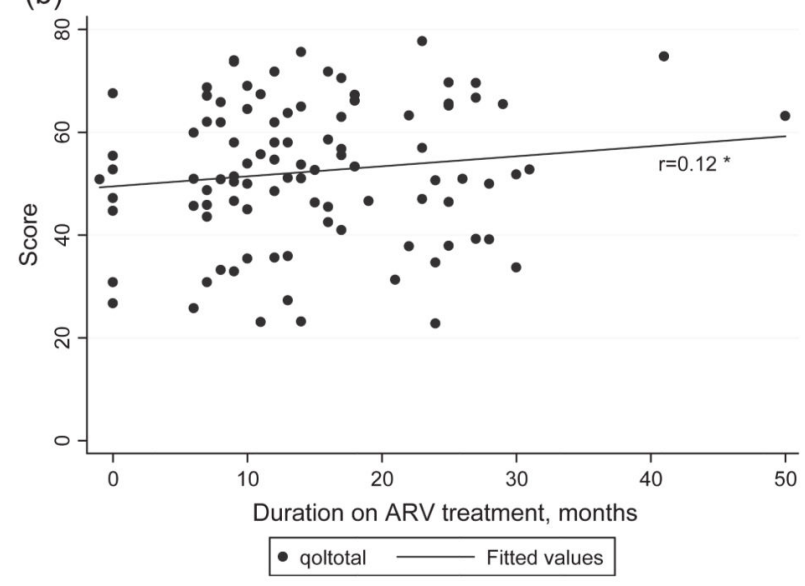

Figure 2.

(a) Physical health summary (PHS) and mental health summary (MHS) scores as functions of duration on antiretroviral (ARV) treatment for the treated group only $(n=96)$. *Spearman rank correlation coefficients, both $p>0.05$. (b) Mean QOL scores as a function of duration on antiretroviral (ARV) treatment for the treated group only $(n=96)$. *Spearman rank correlation coefficients, $p>0.05$. 


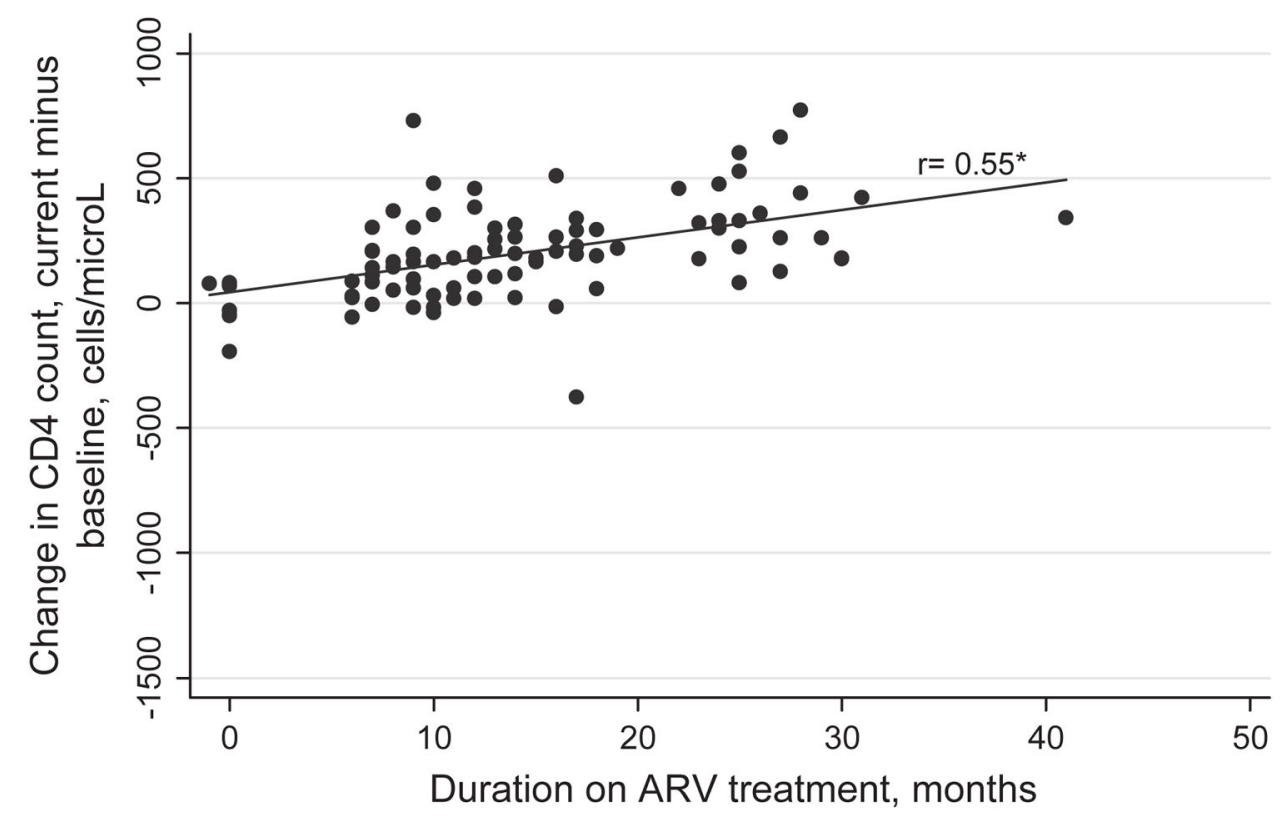

- cd4change Fitted values

Figure 3.

Change in CD4 counts (current minus baseline) as a function of duration on antiretroviral $(\mathrm{ARV})$ treatment for the treated group only $(n=96)$. *Spearman rank correlation coefficients, $p<0.0001$. 


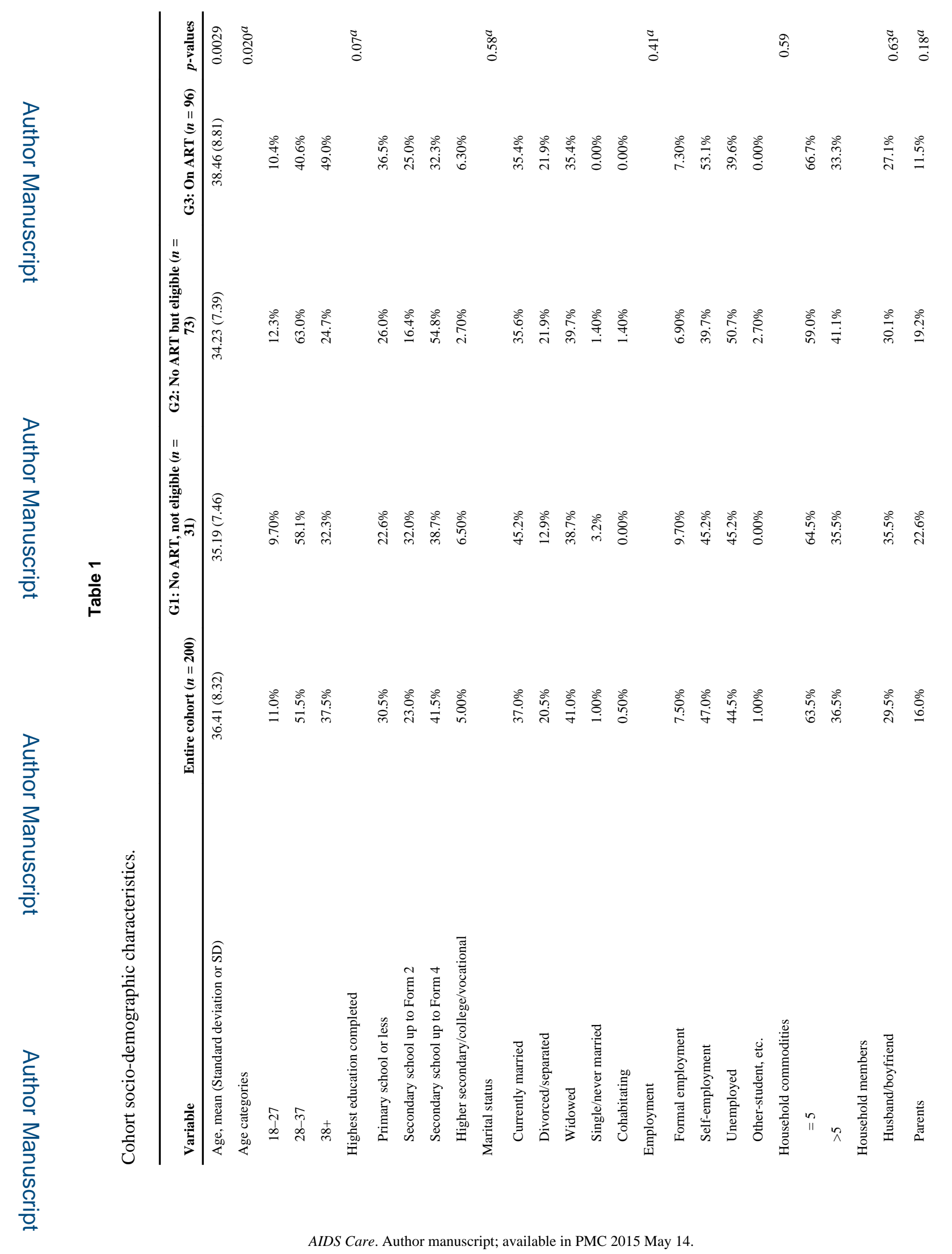




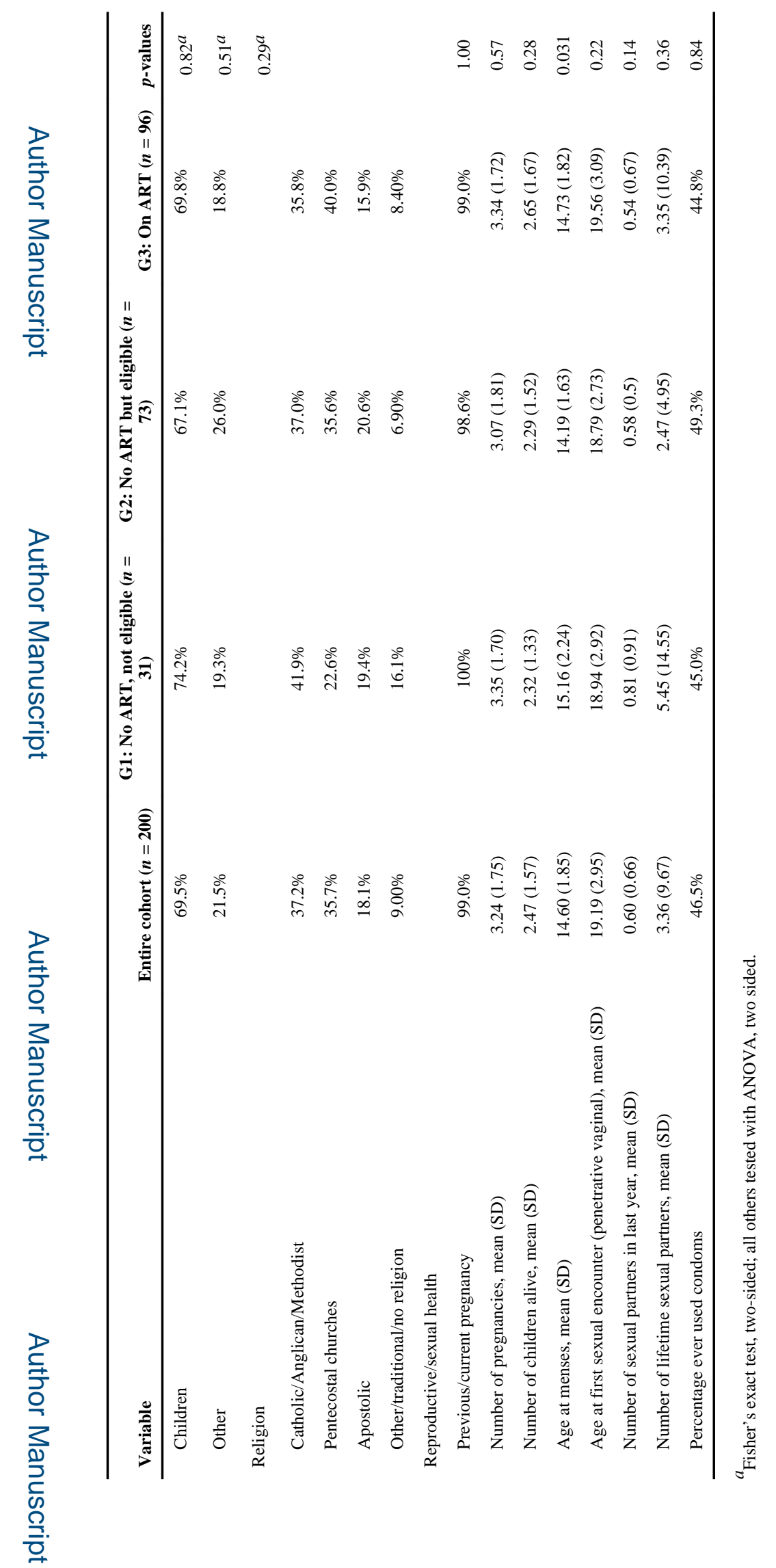

AIDS Care. Author manuscript; available in PMC 2015 May 14. 


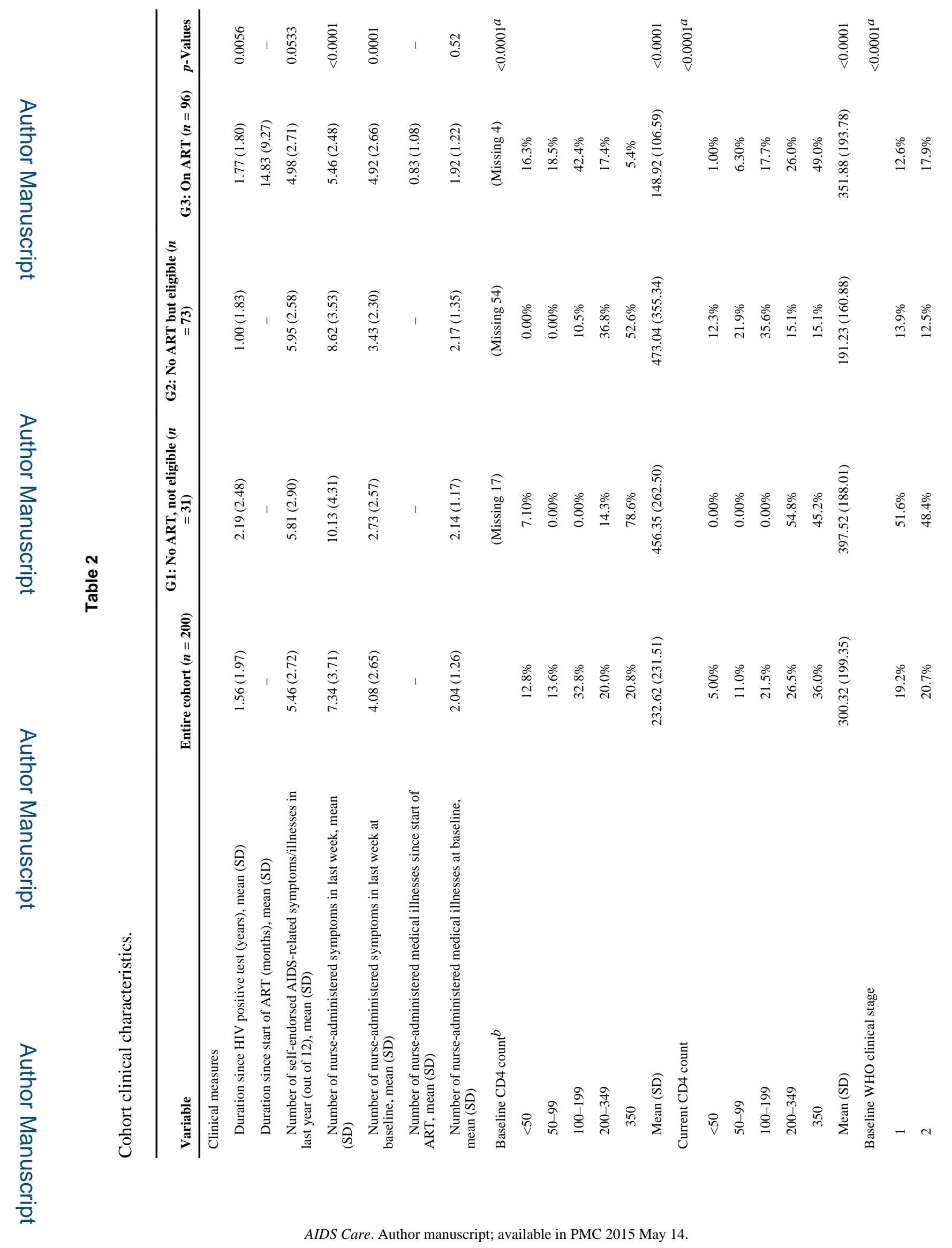


Patel et al.

Page 17

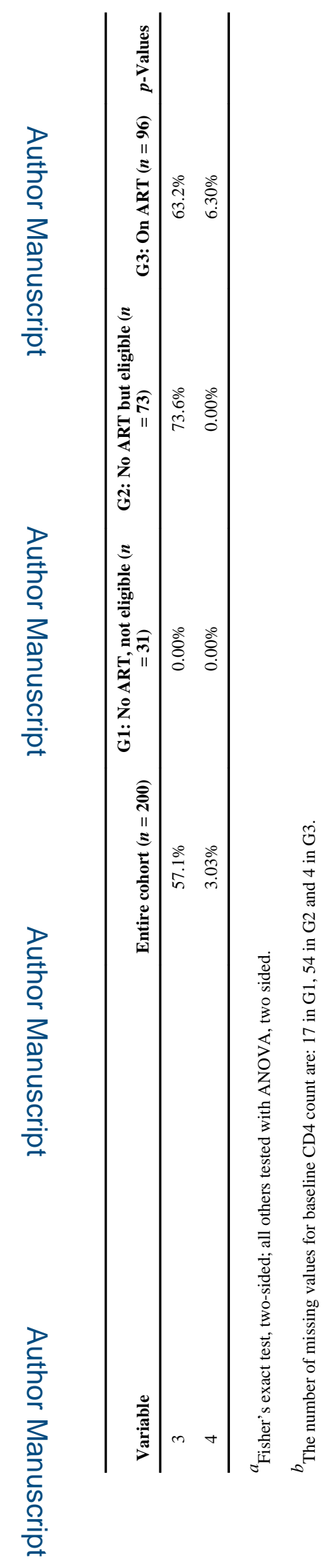

AIDS Care. Author manuscript; available in PMC 2015 May 14. 


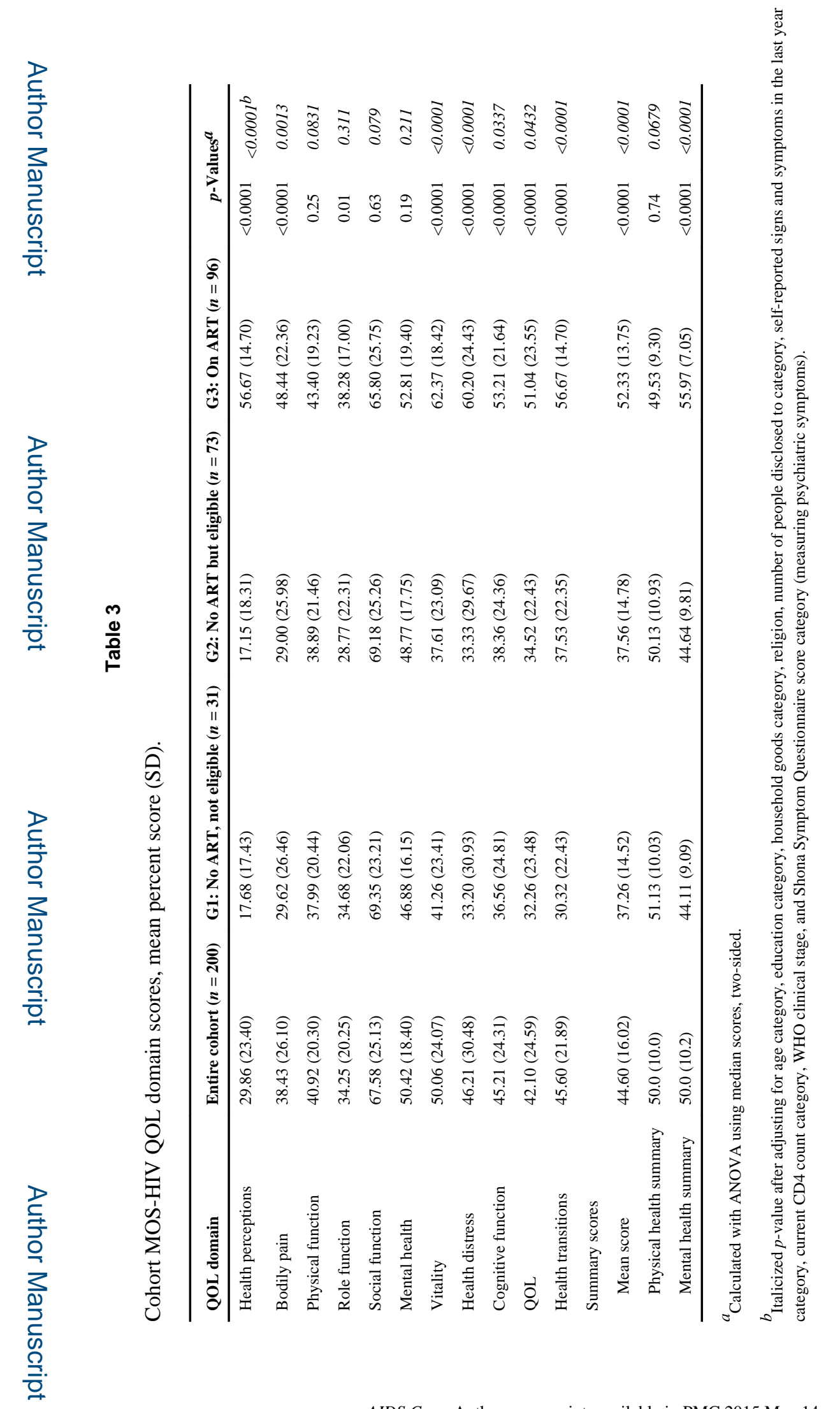

AIDS Care. Author manuscript; available in PMC 2015 May 14. 


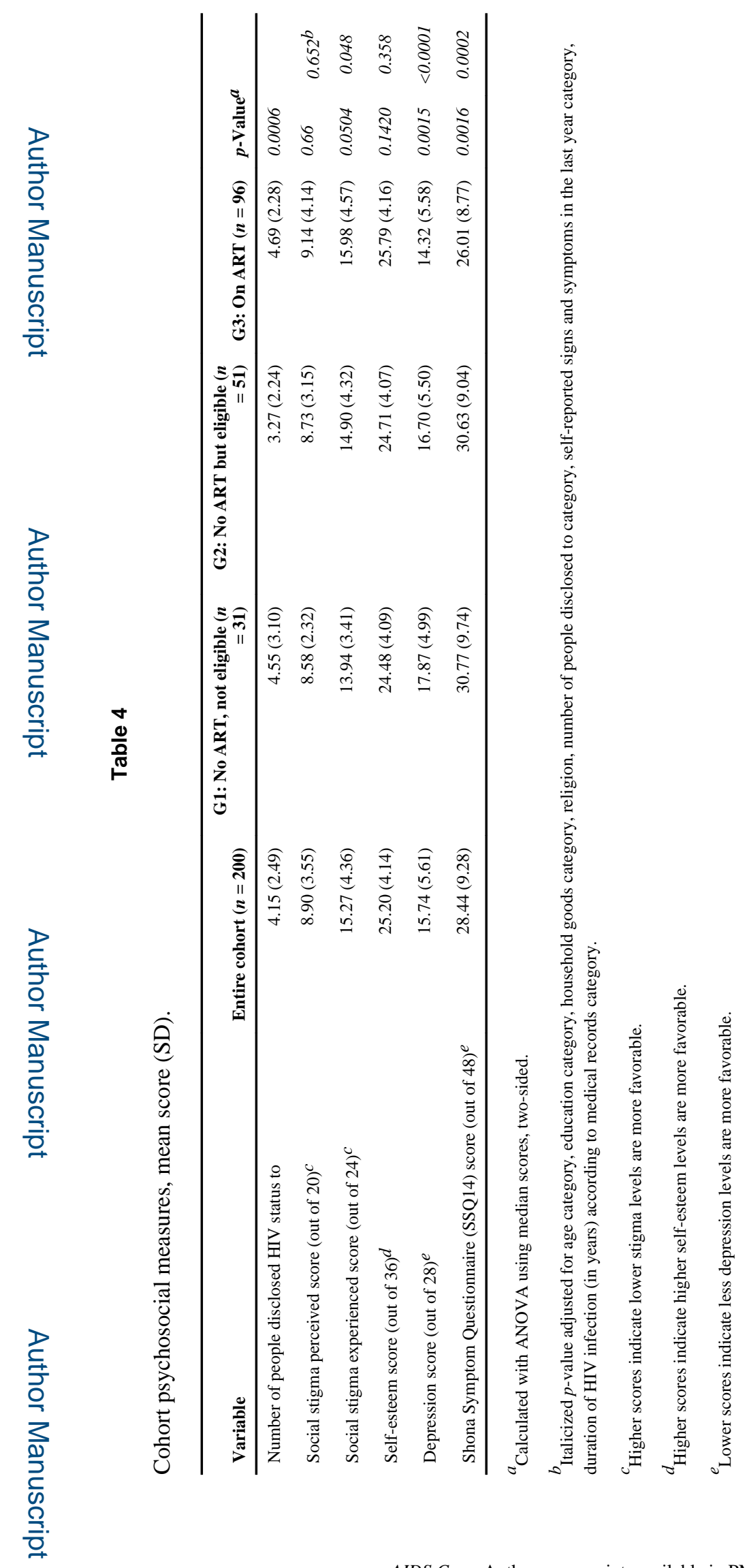

AIDS Care. Author manuscript; available in PMC 2015 May 14. 
Table 5

Potential predictors of QOL summary scores ${ }^{a}$ ( $p$-values only).

\begin{tabular}{lccc}
\hline Variable & Mean QOL score & PHS score & MHS score \\
\hline Treatment groups & $<0.0001$ & 0.86 & $<0.0001$ \\
Age categories & 0.34 & 0.44 & 0.19 \\
Education categories & 0.018 & 0.37 & 0.063 \\
Household goods categories & 0.062 & 0.69 & 0.014 \\
Religion & 0.71 & 0.43 & 0.24 \\
Number of self-endorsed AIDS-related symptoms/illnesses in the last year & 0.0002 & $<0.0001$ & 0.10 \\
Current CD4 count categories & 0.0007 & 0.32 & 0.0010 \\
Baseline WHO clinical stage & 0.045 & 0.0036 & 0.38 \\
Duration since first HIV-positive test, categories & 0.91 & 0.25 & 0.37 \\
Number of people disclosed HIV status to, categories & 0.97 & 0.28 & 0.44 \\
Shona symptom questionnaire score categories & $<0.0001$ & 0.54 & $<0.0001$ \\
\hline
\end{tabular}

${ }^{a}$ Conducted using a backward stepwise linear regression model. 